\title{
SOME CHARACTERISTICS OF DYNAMIC SPECTRA OF SOLAR BURSTS
}

\author{
F. T. HADDOCK \\ University of Michigan, Ann Arbor, Michigan, U.S.A.
}

The sun has been observed daily at the University of Michigan during the last year with three sweep-frequency receivers covering the 100 to $580 \mathrm{Mc} / \mathrm{s}$ band three times a second. The output is displayed as an intensity-modulated line on a precision cathode-ray tube that is photographed on a $35-\mathrm{mm}$ film moving about one centimeter per minute, thereby producing a frequency-time record with solar intensities recorded as variations in photographic density. The combination of the film characteristic and the logarithmic response of the IF amplifier permits the recording of a wide range of intensities in greater detail than before. A number of typical radio events associated with solar flares have been obtained; a typical sequence is a short group of intense type III bursts (fast drifts) followed within minutes by a type II burst (slow drifts) lasting 10 to 30 minutes, and followed by a type I noise storm, with or without a continuum increase, continuing for hours or days. The type I event is usually confined to frequencies below about $200 \mathrm{Mc} / \mathrm{s}$, whereas the type III and type II (bursts) have been recorded up to $580 \mathrm{Mc} / \mathrm{s}$.

\section{TYPE II BURSTS}

Several type II bursts obtained during the first month of operation showed dynamic spectra with very fine detail, appearing as if they were composed of numerous fast-drift bursts similar to the type III bursts but limited in frequency to a band of 10 to $50 \mathrm{Mc} / \mathrm{s}$ [1]. Superimposed on these narrow-band fast-drift bursts appeared an increase in continuum emission, also limited in frequency and drifting to lower frequencies with time. Furthermore, occasionally superimposed on the type II bursts are groups of U-bursts, to be described later, groups lasting for less than $\mathbf{3 0}$ seconds, and all bursts of a group having approximately the same reversing frequency. The U-bursts appear in groups similar to type III bursts and have other characteristics in common with them. These facts suggest that fast drifts, limited in frequency range, may be the principal component of a composite spectrum that has been recorded in the past as a single simple event. On a number of occasions records of type II bursts show a wavy or varying drift in frequency over a range of about $20 \mathrm{Mc} / \mathrm{s}$ and drifting at a rate of 20 to $100 \mathrm{~km} / \mathrm{second}$, on a basis of an undisturbed coronal model.

Wild and co-workers [2] in Sydney have confirmed the existence of fine detail in the type II burst and have noted a detailed structure referred to by 
them as a herringbone pattern. No clear evidence of this pattern has been noted on the Michigan records, although it should be pointed out that the Sydney records were obtained at lower frequencies.

A number of records show detailed features of a type II burst having a one-to-two frequency relationship. These features are sometimes narrow band, 20 to $50 \mathrm{Mc} / \mathrm{s}$ wide, and lasting 10 to 100 seconds. Sometimes they are structureless "continuum" features lasting for 10 to 20 seconds and also restricted in bandwidth to 20 to $50 \mathrm{Mc} / \mathrm{s}$. This suggests that the mechanism responsible for the type III and type II bursts is closely associated with a continuum emission that does not involve the synchrotron process because of its narrow bandwidth. However, it may be emission from gyrating electrons in magnetic fields or nonlinear plasma oscillations.

One has the impression that a type II burst indicates the existence of an outward drifting magnetic field, expanding with a velocity of 500 to 2000 $\mathrm{km} / \mathrm{second}$, and creating regions potentially capable of generating radio waves by plasma oscillations or gyrating electrons when excited by fast streams of charged particles or shock waves. If this were so then the type III and type II bursts could be generated by the same type of high-speed exciting agency. The type III bursts could be due to particle streams or shock waves sufficiently strong to create radio bursts without the perturbation of the corona, whereas the type II events require special conditions of magnetic field, because of the weaker intensity of the particle streams or shock waves, for example.

The expanding magnetic field would carry along with it protons and electrons at its expanding velocity. These particles could cause the terrestrial events presumed to follow a flare by 1 to 3 days. Type I noise storms are known to originate well above the plasma-frequency level in the solar corona, and the existence of circular polarization in the noise bursts requires at least 50 gauss fields at a large distance from the solar surface. As will be mentioned later, the type IV burst also requires a strong magnetic field at a great distance from the solar surface and it frequently follows a type II burst.

\section{TYPE IV BURSTS}

At the 1957 General Assembly of URSI in Boulder, Denisse described work by Boischot [3] that identified a new class of solar bursts, which they classified as type IV. It was described as a broad-band continuum increase, free of bursts, and lasting from 20 minutes to a few hours. With a narrow fan beam at $169 \mathrm{Mc} / \mathrm{s}$ Boischot found that the emitting region was as large as one-third of the solar diameter and that it extended to several solar radii above the sun's surface. On the basis of these characteristics Denisse put forth the hypothesis that the type IV event was due to synchrotron radiation.

On September 12, a record was obtained at the University of Michigan of a typical sequence of events associated with a solar flare but with a general broad-band brightening following the onset of the type II burst, two or three minutes after the initial type III burst group. This broad-band continuum was intense and free of detailed structure. It lasted 150 minutes. An inter- 


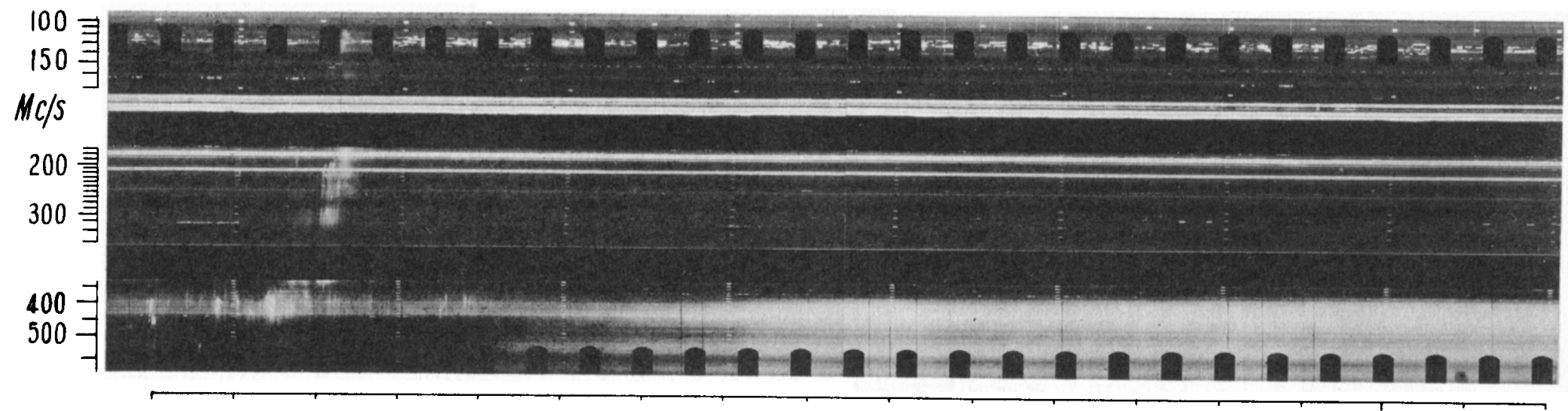

FIG. 1. An intensity-modulated recording of a type IV radio-burst spectrum beginning about $14 \mathrm{~m} 20^{\mathrm{m}} \mathrm{UT}$. $14^{\mathrm{h}} 30^{\mathrm{m}} \mathrm{UT}$.

September 13. This event lasted 90 minutes. solid and broken solid and broken, are man-made interfering signals. The vertical columns of dashes are frequency and time markers. The type IV burst is the brightening between the frequencies of 350 and $580 \mathrm{Mc} / \mathrm{s}$. It begins about 2 minutes after the groups of type III and U-burst groups at the left.

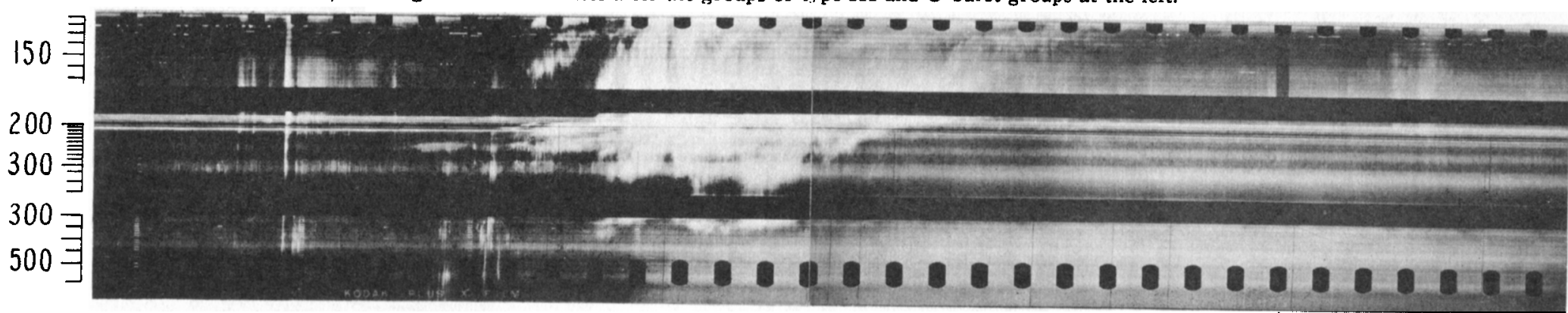

$17^{\text {h }}$ U.T.

Fig. 2. A recording of a sequence of dynamic radio spectra at the time of a solar flare on 1957 October 20 . An initial group ot type III bursts occurred at $16^{\text {b } 48 \text { m }}$ U.T., followed in three minutes by the onset of a type II burst on the two lower frequency bands and by the onset of a type IV burst on the high-frequency band. The latter burst extended to the lower-frequency bands within a few minutes. It lasted 45 minutes. 
esting feature of this broad-band continuum was the apparent decrease in emission below about $320 \mathrm{Mc} / \mathrm{s}$. Since this decrease in intensity occurred at the low end of the high-frequency sweep receiver it is partially, or even largely, caused by instrumental effects. On the next day, however, another flare-associated radio event, without the appearance of the type II burst, but with a group of type III and U-bursts, was followed within two minutes by a continuum increase at frequencies above $360 \mathrm{Mc} / \mathrm{s}$ and with almost no apparent increase in brightness below $360 \mathrm{Mc} / \mathrm{s}$. Fig. 1 is a photograph of this record. The high-frequency sweep receiver extended down to below this abrupt change of intensity. We cannot attribute all of this change to the instrumental effects although gain variations do account partially for the abruptness of the low-frequency cutoff on this type IV event.

On October 20 , beginning about $16^{\mathrm{h}} 47^{\mathrm{m}} \mathrm{U} . \mathrm{T}$. another sequence of radio events associated with a solar flare was recorded. Fig. 2 is a photograph of the radio spectrum of this event. The type II burst began about 3 minutes after this initial type III burst. The high-frequency limit of the type II burst was about $360 \mathrm{Mc} / \mathrm{s}$. A general increase in continuum on the high-frequency receiver began about 4 minutes after the initial type III burst group. This continuum was relatively free of detail and lasted about 45 minutes; it could be seen on the middle- and low-frequency receivers but the high-frequency intensity was greater. In this event there was no evidence of a sharp lowfrequency cutoff. Measurements of polarization at $200 \mathrm{Mc} / \mathrm{s}$ by Cohen at Cornell University indicate that the polarization of this type IV burst was 30 per cent left-hand circular.

\section{U-TYPE BURSTS}

A detailed analysis of U-type bursts obtained during the first few months of observation was made by Takakura and will appear as a separate paper. The following is largely from that paper. On 1957 September 3, at about $12^{\mathrm{h}} 15^{\mathrm{m}}$ U.T. three simultaneous bursts were recorded with U-type spectra having a duration of a few seconds with their low-frequency reversing points at 130,150 , and $375 \mathrm{Mc} / \mathrm{s}$. Between 130 and $120 \mathrm{Mc} / \mathrm{s}$ there existed a steady interference signal that would obscure a few $\mathrm{Mc} / \mathrm{s}$ of the low-frequency limit of the $130 \mathrm{Mc} / \mathrm{s} \mathrm{U}$-burst; the lower frequencies of this burst could also be absorbed since they are near the critical-frequency level in the solar corona. The following facts lend support to the assumption that these bursts are harmonics of the same event: $(a)$ the time of the "reversing "frequency on these three U-bursts was the same within the observational uncertainty of one second; (b) the "outward," or decreasing-frequency branch, of the U-burst was more intense on all three bursts than the "returning branch"; $(c)$ the over-all duration was the same; and $(d)$ there was an exact two-tothree frequency ratio between the two higher-frequency bursts. Since the high-frequency burst appears to be more intense than the low it may be that the fundamental frequency is subjected to more absorption or scattering than the harmonics; its "escape cone" may be narrow because of its closeness 
to the critical frequency level. This would make the fundamental frequency burst detection a rare event and suggests that most recorded U-bursts are second harmonic bursts or higher.

The 40 U-bursts analyzed exhibited a remarkable uniformity of duration, which was independent of the "reversing" frequency of the U-burst. There is a suggestion, however, that on a frequency below $170 \mathrm{Mc} / \mathrm{s}$ there are a few U-bursts that are much longer in duration and that may be another class of events. U-bursts occur in groups of two to ten, which last for 10 to 30 seconds. The same typical duration has been noted for type III bursts. Both types also have the same maximum rates of frequency-drift and in the few cases studied they have about the same percentage of random polarization.

Takakura and the author have proposed that U-bursts are generated by charged particle streams that do not have sufficient outward velocity to overcome the magnetic field embedded in them. The particles reach a maximum height above the sun which depends upon their initial kinetic energy, and are pulled back toward it with a maximum velocity comparable to that at which they were shot out. Their angular momentum prevents a simple radial trajectory. Rather large magnetic-field intensities are required to account for the height and velocities involved, but the values are of the same order as the field strengths required by Takakura to account for the type IV bursts [4]. The energies involved are less than the total energy in a solar flare.

It is a pleasure to thank Dr. T. Takakura for valuable discussion and analysis of data, Mr. M. Winsnes, Mr. J. W. Kuiper, and Mr. J. O. McDermott for operation of the equipment.

\section{REFERENCES}

[1] Haddock, F. T. Proc. I.R.E. 46, 3, 1958.

[2] Wild, J. P., Sheridan, K. V., and Trent, G. H. Paper 32.

[3] Boischot, A. C.R. 244, 1326, 1957.

[4] Takakura, T. Paper 101.

\section{Discussion}

Pecker: When positional measurements of U-bursts are made, are the source positions at a given frequency the same for ascending and descending branches?

Roberts: No.

de Jager: Whereas Dr. Boischot needed a field of only some gauss to explain the type IV emission, Professor Haddock seem to favor some hundreds of gauss.

Haddock: At a later session Dr. Takakura will present a theory of type IV bursts that requires a magnetic field of the order of 350 gauss.

Thompson: I should like to show two examples of peculiar U-bursts 
recorded with the sweep-frequency equipment at Fort Davis, Texas. The first, which occurred 1957 April $17^{\mathrm{d}} 11^{\mathrm{h}} 52^{\mathrm{m}}$ U.T., showed the usual fast frequency decrease to the turn-over point, and then split into three separate parts. The possible explanation involves splitting the moving disturbance by the magnetic fields of a complex spot group. The second record, obtained 1957 May $28^{\mathrm{d}} 16^{\mathrm{h}} 57^{\mathrm{m}}$ U.T., shows two U-bursts that occurred simultaneously. The turn-over frequencies are approximately 115 and $160 \mathrm{Mc} / \mathrm{s}$.

Fokker: As to the relation between radio phenomena and geomagnetic storms, I should like to remark that about 60 per cent of the sudden commencements of geomagnetic storms can safely be ascribed to a particular flare-associated radio event, which in virtue of its integrated intensity belongs to the greatest category of outbursts, from meter to centimeter wavelengths. Most of these important radio events seem to belong to type IV, not to type II. They precede the SC by one or two days. Furthermore, I want to stress that one should be very careful in drawing conclusions about a physical relationship between geomagnetic storms and a particular type of event from superposed epoch diagrams. As a matter of fact, phenomena like flares, bursts of types II, III, and IV, and geomagnetic storms, all tend to occur in periods of enhanced solar activity. As to this circumstance we made a test by considering the sudden commencements that preceded and followed a particular flare within 5 days. The SC's following flares appeared to outnumber significantly the preceding ones, even when the flares considered were selected at random from the Meudon list, without any reference to their importance or to associated radio events! 